\title{
Preliminary observations on the role of magnetic resonance imaging for polymyositis and dermatomyositis
}

\author{
LEE D KAUFMAN,' BARRY L GRUBER!' DAVID P GERSTMAN , AND ALAN \\ T KAELL'
}

From the 'Department of Medicine and the Division of Allergy, Rheumatology, and Clinical Immunology, State University of New York at Stony Brook, Stony Brook, NY; and ${ }^{2}$ Suffolk Magnetic Resonance Imaging, $P C$, Smithtown, NY

SUMMARY The potential use of magnetic resonance imaging (MRI) for inflammatory muscle disorders was evaluated in 13 patients with polymyositis and dermatomyositis. Abnormalities in signal intensity $(p=0.0076)$ and fat replacement $(p=0.0177)$ were identified and correlated significantly with clinical disease activity. In addition, MRI was useful in directing muscle biopsy of selected abnormal areas.

Magnetic resonance imaging (MRI) is a noninvasive technique, the applications of which are rapidly increasing. Recent studies have shown that normal muscle can be differentiated from myopathic tissue by proton imaging.' We explored the potential use of this modality in the diagnosis and management of patients with polymyositis and dermatomyositis and report abnormalities that correlate with clinical activity.

\section{Patients and methods}

Thirteen patients meeting clinical criteria ${ }^{2}$ for polymyositis and dermatomyositis were selected for study, and five normal volunteers matched for age were chosen as controls.

Transaxial and coronal sections of the shoulders and thighs were obtained on each patient using a $1 \cdot 0$ $T$ superconducting magnetic resonance system operated at $0.5 \mathrm{~T}$ (Picker International, model Vista 2055). For thigh assessment the pulse sequences used were a repetition time of $700 \mathrm{~ms}$ and an echo time of $40 \mathrm{~ms}$; for shoulder assessment we used a repetition time of $550 \mathrm{~ms}$ with an echo time of $26 \mathrm{~ms}$. Sections $1 \mathrm{~cm}$ thick were obtained for all projections. $T_{1}$ weighted images were obtained in all studies as the addition of $T_{2}$ weighted sequences initially offered no advantage.

Accepted for publication 26 March 1987.

Correspondence to Dr Lee D Kaufman. HSC-T-16-140. Division of Allergy. Rheumatology. and Clinical Immunology. Statc University of New York at Stony Brook. Stony Brook. NY 11794. USA.
Each study was arbitrarily evaluated with a quantitative four point scale for the degree of abnormality, with 0 being normal and 3 severe. Images were graded for the following parameters: focal $v$ diffuse abnormalities, signal intensity, fat replacement, and atrophy in a blinded fashion. Disease activity was determined by the presence or absence of abnormalities in muscle enzymes (creatine phosphokinase, aldolase), electromyography (EMG) findings, muscle biopsy, and proximal muscle weakness. Active disease was defined as the presence of at least three out of these four criteria. All patients with dermatomyositis had the typical cutaneous findings. Statistical analysis was by unpaired $t$ test.

\section{Results}

Five patients had polymyositis and eight patients had dermatomyositis. Their ages ranged from 12 to 77 years (mean 44.7$)$ and the ages of the controls from 33 to 50 years (mean 38.2). Eight patients met the criteria for clinically active disease and five patients were inactive at the time of MRI.

Transaxial sections of the thighs were more easily standardised for comparison than were coronal views of the thighs or either view of the shoulders. The mean scores for each selected parameter in patients with active $v$ inactive disease were as follows: $2.25 v 0.6(\mathrm{p}=0.0076)$ for signal intensity: $2.5 v 0.8(\mathrm{p}=0.0177)$ for fat replacement; and $2.25 v$ $0.8(p=0.0584)$ for atrophy. These data demonstrate a clear difference between patients with active or 


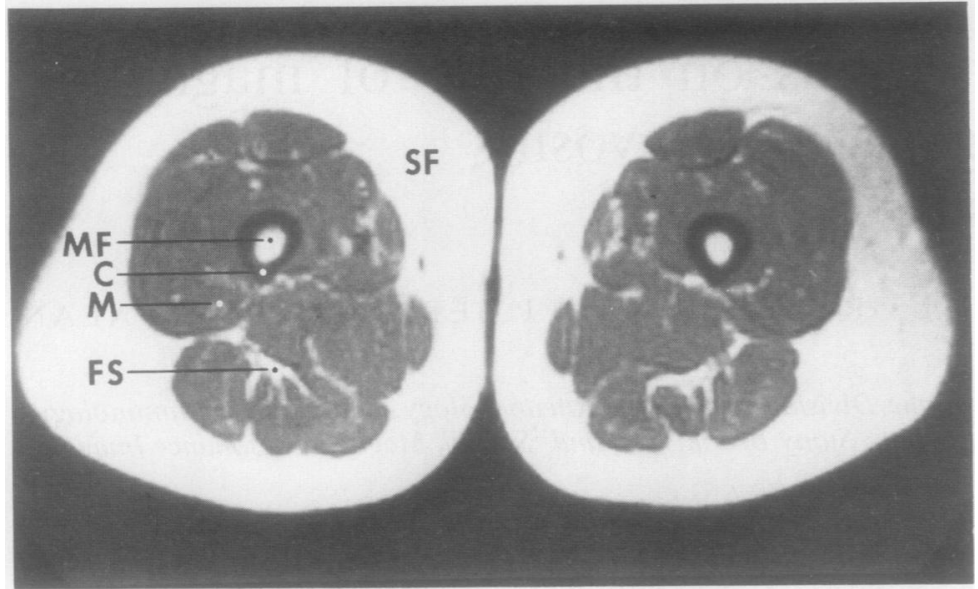

Fig. 1 Transaxial section through the thighs of a normal volunteer. Femoral marrow fat (MF), cortical bone (C). normal signal intensity of muscle (M), fibrous septae (FS). and subcutaneous fat (SF) are all easily distinguished from each other.

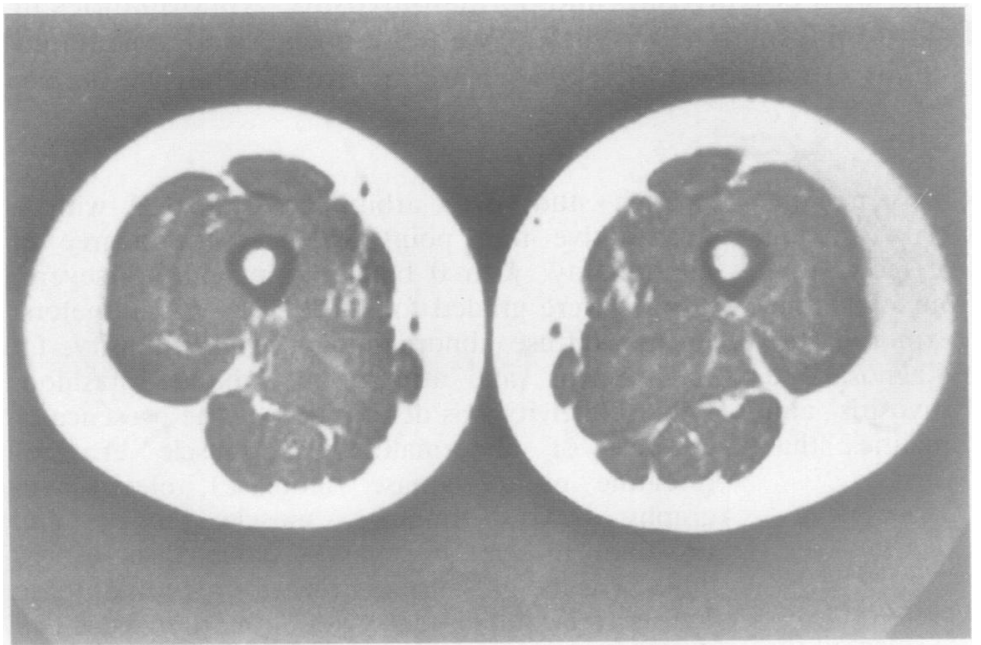

rig. 2 Transaxial section through the thighs of a patient with inactive polymyositis resembling the normal MRI findings delineated in Fig. I (score ()).

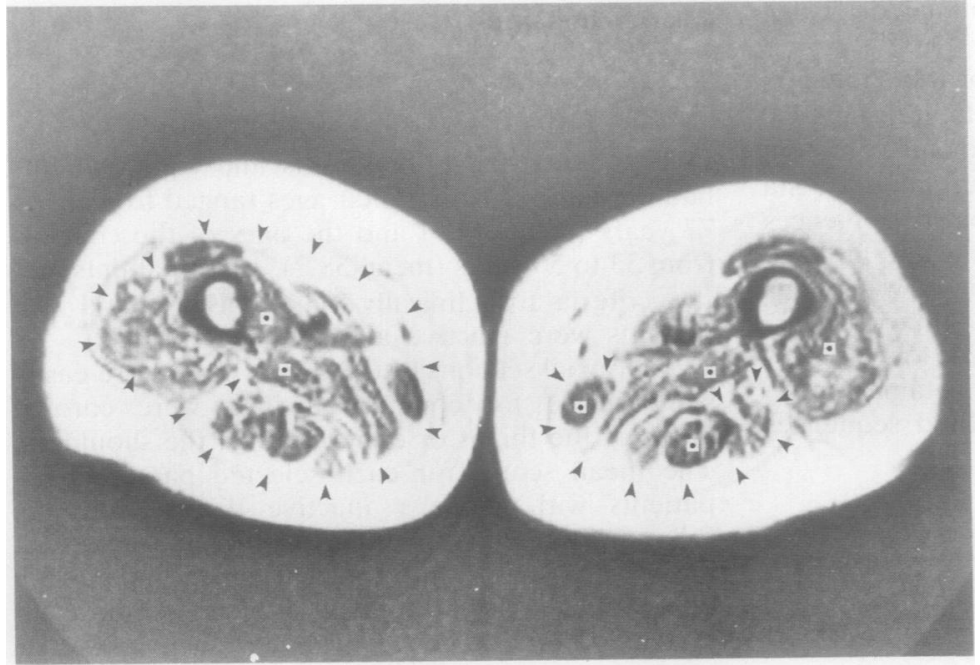

Fig. 3 Transaxial section through the thighs of a patient with active polymyositis demonstrating 'fat' replacement of muscle groups $(\rightarrow)$, decreased signal intensity of muscle fibres ( $\square$ ), and generalised atrophy (score 3). 


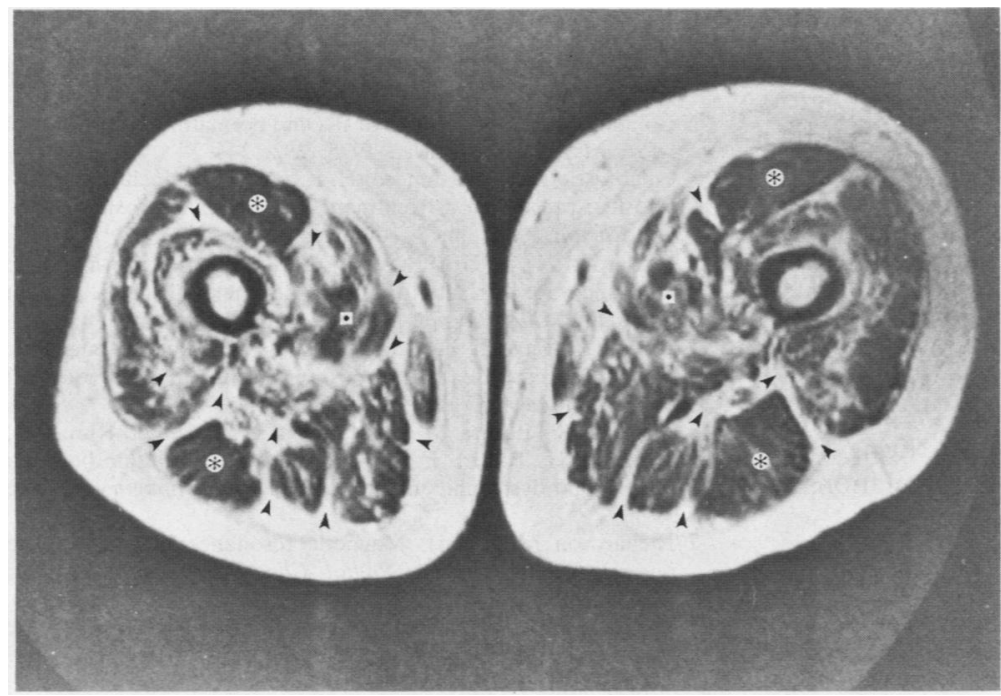

Fig. 4 Transaxial section through the thighs of a patient with inclusion body myositis. Focal abnormalities of replacement $\rightarrow$ ) and decreased signal intensity of muscle (๑) are interspersed with normal appearing muscle bundles $\left({ }^{*}\right)$.

inactive disease. Fig. 1 shows the MRI findings in a normal overweight volunteer. Subcutaneous fat is easily distinguished from muscle bundles and intervening fibrous septae. Fig. 2 shows the similar findings in a patient with inactive polymyositis. These studies are contrasted with the extreme differences one can observe in a patient with active myositis (Fig. 3). Only one patient of the 13 studied demonstrated focal abnormalities with MRI (Fig. 4). This segmental change led to rebiopsy of an involved area which showed inclusion body myositis. ${ }^{3}$

\section{Discussion}

Recent studies have demonstrated the use of nuclear magnetic resonance (NMR) in detecting structural and functional abnormalities in various neuromuscular processes. ${ }^{1+5}$ Specifically, probes using ${ }^{31} \mathrm{P}$ and ${ }^{1.3} \mathrm{C}$ NMR spectroscopy have been useful in studying metabolic changes in muscle disease which relate to clinical fatigue, such as the rate of glycolysis, fluctuation of $\mathrm{pH}$, and adenosine triphosphate utilisation. ${ }^{+}$

The application of proton NMR spectroscopy has recently been employed to differentiate between normal and dystrophic muscle in an avian model of muscular dystrophy as well as in a wide spectrum of neuromuscular diseases in man. ${ }^{5}$ "Furthermore, preliminary evidence has suggested a correlation between the histopathological findings and the degree of abnormalities as assessed by proton NMR spectroscopy. ${ }^{5}$ These variations in spectroscopic findings are felt to be related to changes in myofibrillar water content resulting from the myopathic process.

The clinical application of proton MRI to both axial and appendicular musculoskeletal pathology has been rapidly expanding to include discogenic spinal disease, osteomyelitis, neoplastic disorders of bone and soft tissue, and internal derangements of articular structures, such as trauma to cartilage. ligamentous tears, and inflamed synovial tissue. ${ }^{7-9}$

From these preliminary observations in neuromuscular diseases the current study sought to determine the usefulness of MRI in polymyositis and dermatomyositis. We have shown that imaging by MRI in these disorders discloses abnormalities as assessed by a decreased tissue signal intensity, fat replacement, and atrophy that correlate with the clinical activity of disease. The high intensity signal interpreted as 'fat replacement' in active myositis (Fig. 3) was not seen in overweight normal controls (Fig. 1) and, when biopsy data were available from recently diagnosed active patients, changes consistent with polymyositis were seen, including lymphocyte infiltration, degenerating fibres, and nuclear centralisation. Adipose tissue was not detected. It is of interest that one of our patients had segmental findings by MRI. This patient, unlike the others, initially had equivocal biopsy findings despite classical EMG abnormalities, increased muscle enzymes, and proximal muscle weakness. He had responded to corticosteroids and carried a diagnosis of polymyositis for many years. The focal nature of the myopathic process by MRI led to rebiopsy of an involved area which demonstrated inclusion body myositis. This entity has recently 
been reported to closely mimic polymyositis. ${ }^{3} \mathrm{MRI}$ was therefore useful not only in defining structural aberrations, but also in guiding the muscle biopsy and minimising the sampling error, which can be a significant problem in the diagnosis of these disorders.

The value of MRI in prospectively following up patients with inflammatory muscle disorders is not yet known. Nevertheless, this non-invasive diagnostic tool with, to date, no known biological hazard. may be helpful in the initial evaluation and subsequent follow up of selected patients with polymyositis and dermatomyositis. Long term studies to evaluate histopathological correlations are in progress.

The authors gratefully acknowledge the secretarial assistance of Karen Abramowski, the following physicians for allowing us to study their patients: Dr J Marshall. Dr M Repice. Dr P Chatpar. and Dr R Marcus, and Dr L Arbeit for assistance in the statistical analysis.

\section{References}

1 Murphy W A. Totty W G. Carroll J E. MRI of normal and pathological skeletal muscle. AJR 1986; 146: 565-74.

2 Bohan A. Peter J B. Polymyositis and dermatomyositis (first of two parts) $N$ Engl J Med 1975; 292: 344-7.

3 Calabrese L H. Mitsumoto $\mathrm{H}$. Chou S M. Inclusion body myositis: presenting as treatment resistant polymyositis. Arthritis Rheum 1987; 30: 397-403.

+ Edwards R H T. Griffiths R D. Cady E B. Topical magnetian resonance for the study of muscle metabolism in humans myopathy. Clin Physiol 1985; 5: 93-109.

5 Borghi L. Savoldi F. Scelsi R, Villa M. Nuclear magnetic resonance response of protons in normal and pathologicat human muscles. Exp Neurol 1983; 81: 89-96.

6 Misra L K. Luthra M G. Amtey S R. Elizondo-Riojas G@ి Swezey $\mathrm{S} \mathrm{H}$. Todd L E. Enhanced $\mathrm{T}_{1}$ differentiation betweer

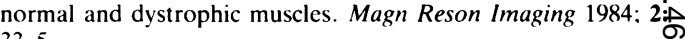
$33-5$.

7 Richardson M L (ed). Magnetic resonance imaging of the musculoskeletal system. Radiol Clin North Am 1986; 24J 137-335.

8 Baker D G. Schumacher H R Jr. Wolf G L. Nuclear magnetie resonance evaluation of synovial fluid and articular tissues $J$ Rheumatol 1985: 12: 1062-5.

9 Adams M E. Li D K B. Magnetic resonance imaging rhcumatology. J Rheumatol 1985; 12: 1038-40. 